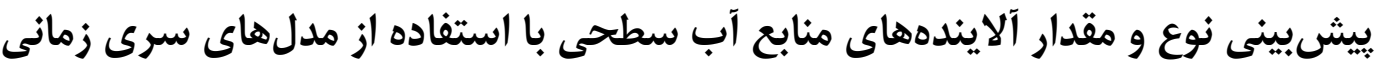

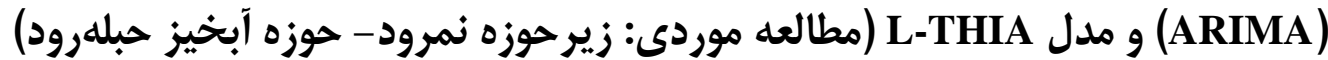

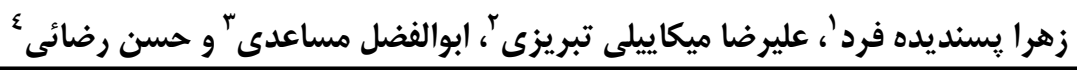 \\ ا- دانشجوى دكترى محيط زيست، ارزيابى و آمايش، گروه محيط زيست، دانشكده شيلات و محيط زيست، دانشكاه علوم كشاورزى و منابع طبيعى گر كان،

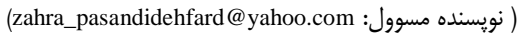 \\ ك و عا- دانشيار و استاديار، كروه محيط زيست، دانشكده شيلات و محيط زيست، دانشكاه علوم كشاورزى و منابع طبيعى كرَّان

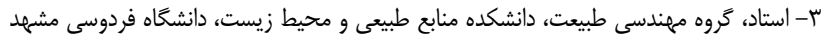

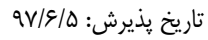 \\ تاريخ دريافت: \\ صفحه:
}

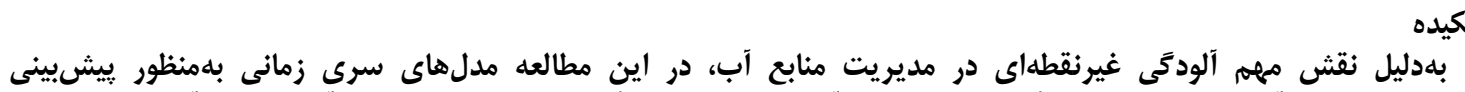

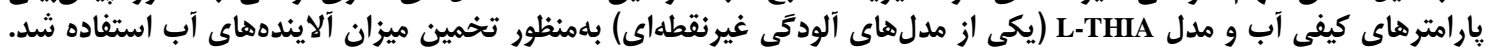

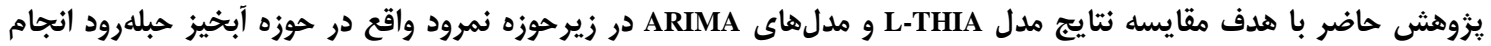

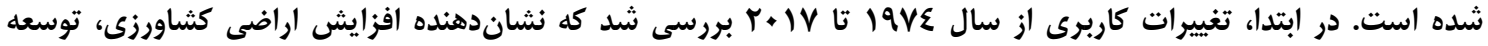

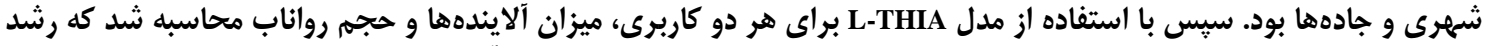

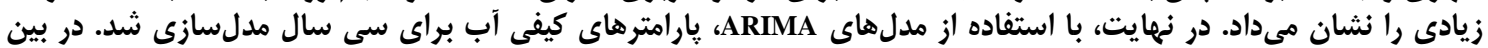

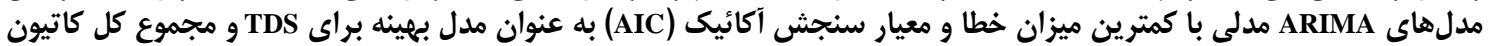

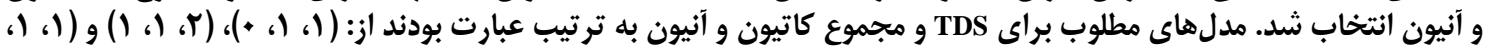

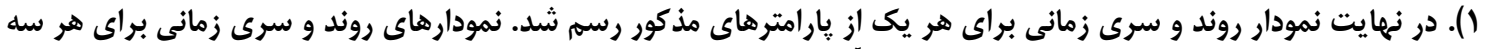

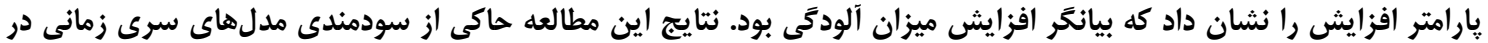

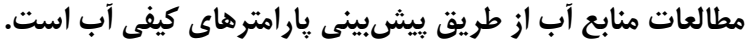

وازههاى كليدى: آلودَّى غيرنقطهاى، يارامترهاى كيفى آب، رواناب، مدل L-THIA، مدلهاى سرى زمانى

نهايت مقدار باركذارى رسوبات معلق و مواد مغذى در شرايط

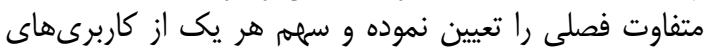

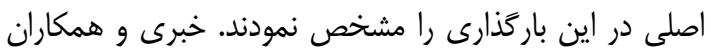

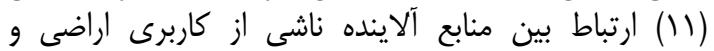

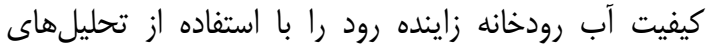

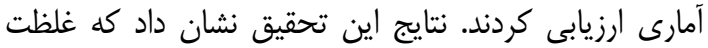

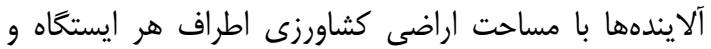

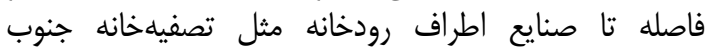

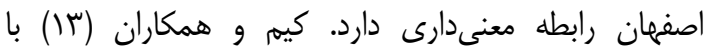

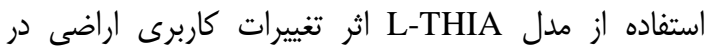

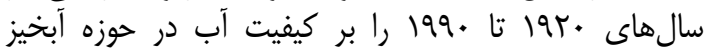

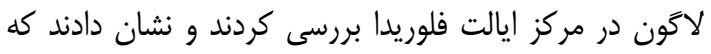

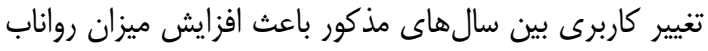

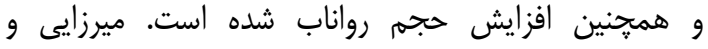

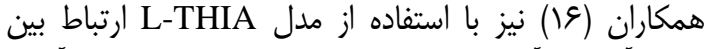

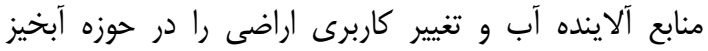

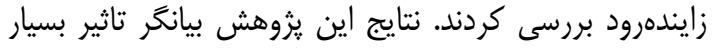

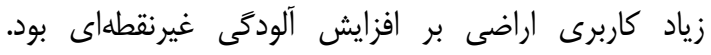

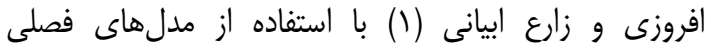

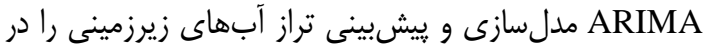

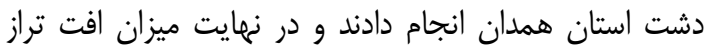

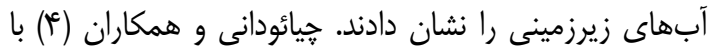

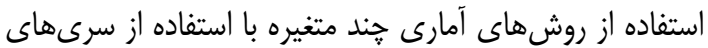

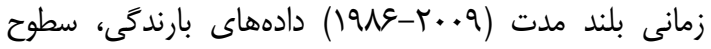

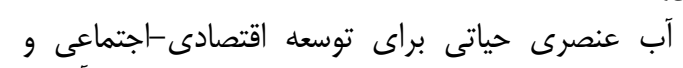

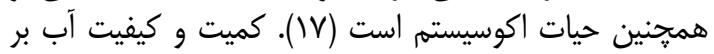

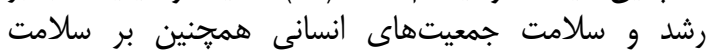

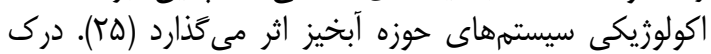

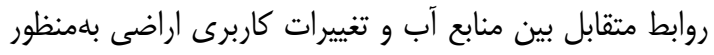

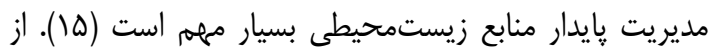

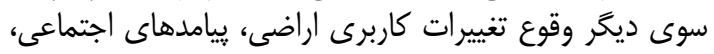

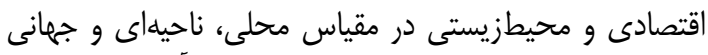

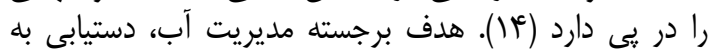

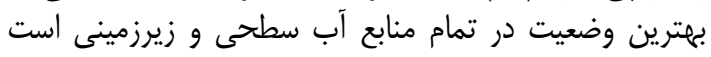

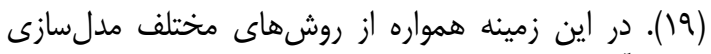

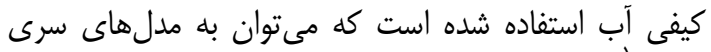

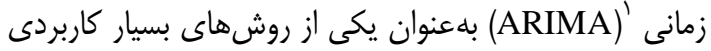

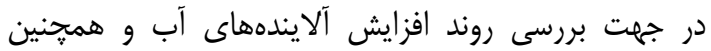
يبيشيينى ميزان رشد آن در آينده اشاره كرد. از آنجا آنجا كه مسئله

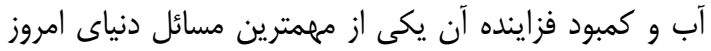

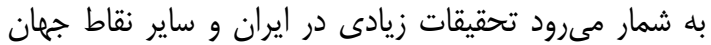

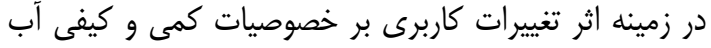

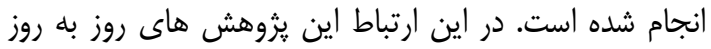

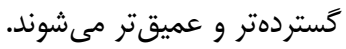

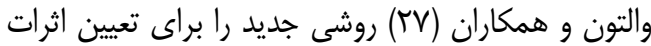

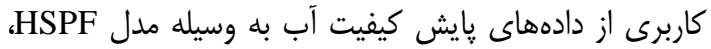
در حوزه آبخيز جان استون در شمال استراليا به كار بردندا دابل در 
زيرزمينى را انجام دادند. ايشان توانستند يك مدل مفهومى

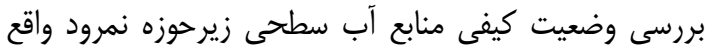
در حوضأ آبخيز حبلدرود، ميزان افزايش آلايندههاى كيفى دئ در سالهاى آتى يِيشينى شودرد

\section{مواد و روشها منطقه مورد مطالعه}

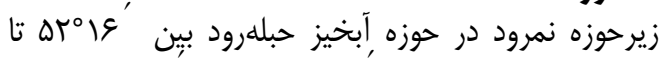
شو

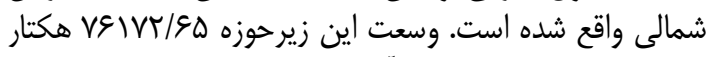

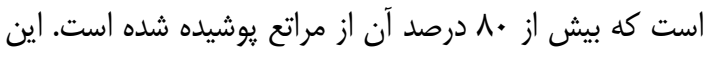

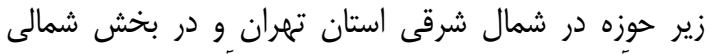

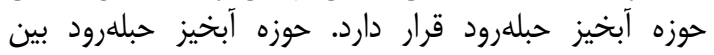

استان هاى تهران و سمنان واقع شده است (شكل (ا) ).

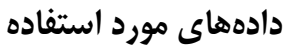

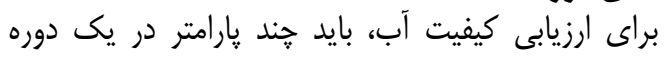

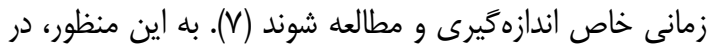

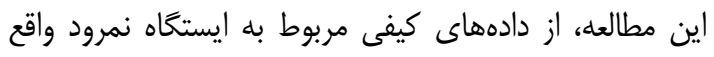

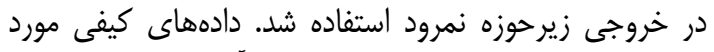

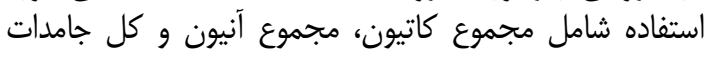

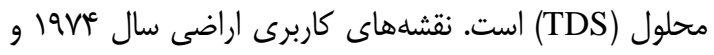

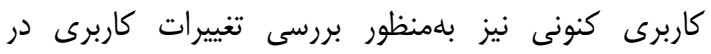
سالهاى مذكور استفاده شد.

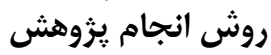

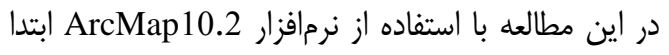

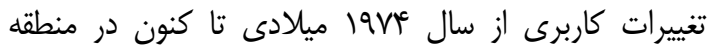

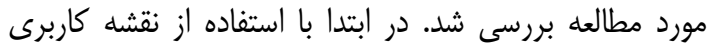

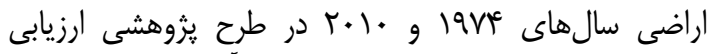

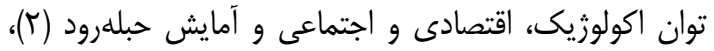

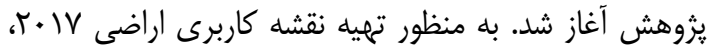

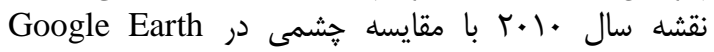

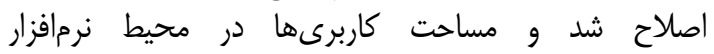

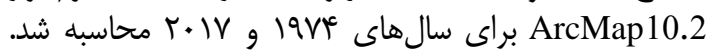

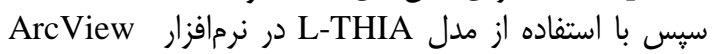
3.2 ميزان آلودگى غيرنقطهاى در سالهاى مذكور بررسى شد.

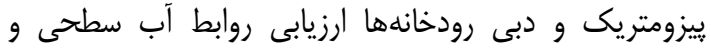

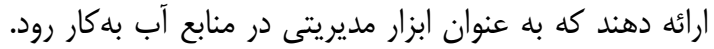

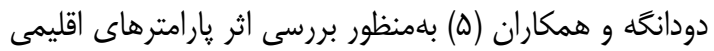

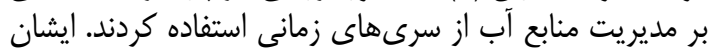

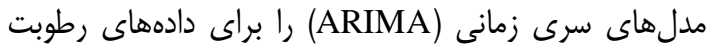

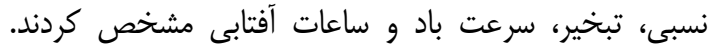

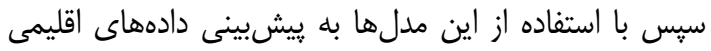

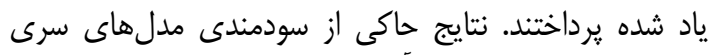

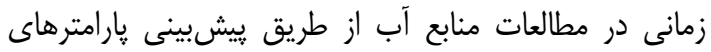

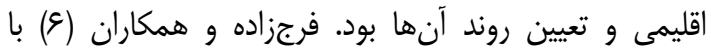

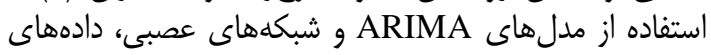

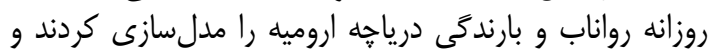

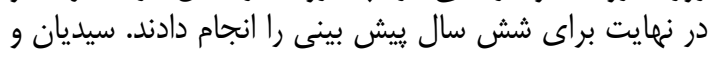

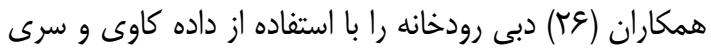

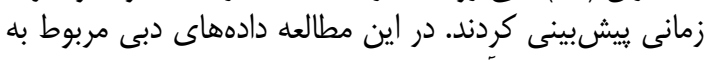

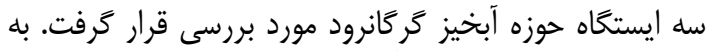

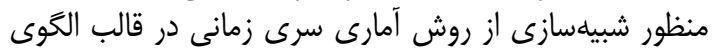

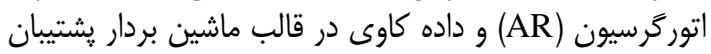
به دو صورت ماهانه و هفتخى استفاده نمودند. نتايج مانيج (SVM)

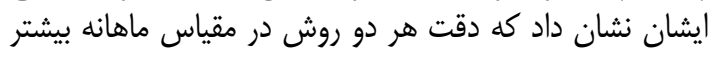

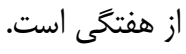

جاويدى صباغيان و شريفى (9) سرى زمانى لهانى •ه ساله

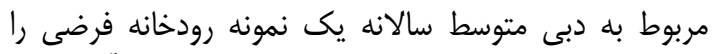

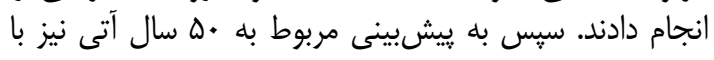

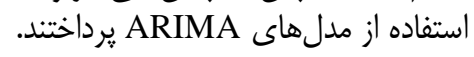

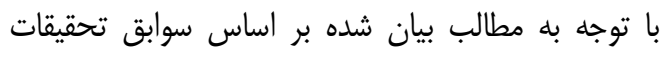

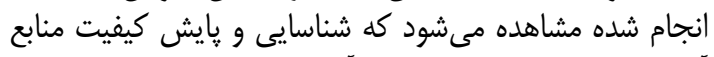

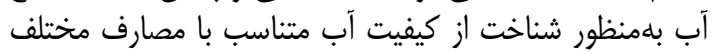

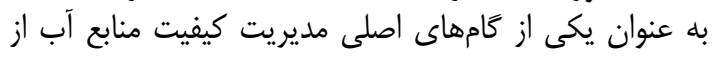

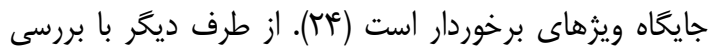

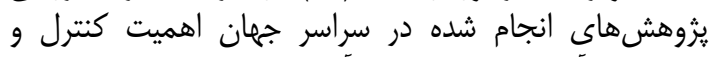

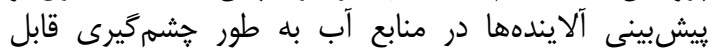

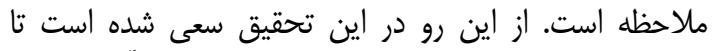

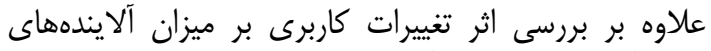

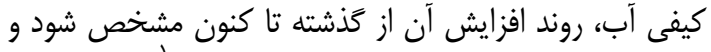

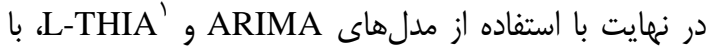

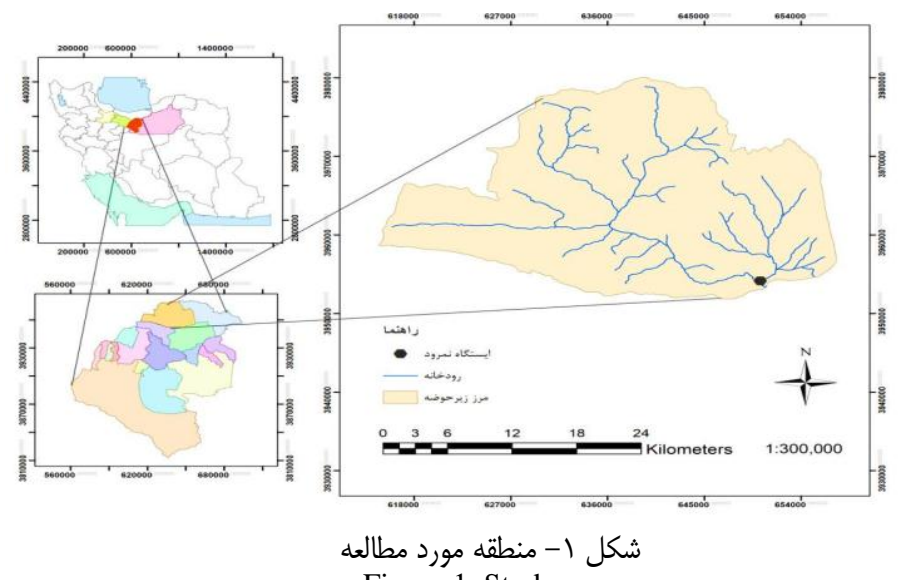

Figure 1. Study area 
منحنى) عمق رواناب و ميزان آلودگى غيرنقطهاى را تعيين

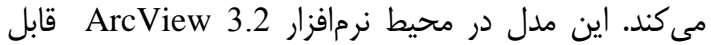

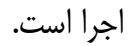

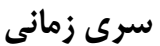
يكى سرى زمانى مجموعه دادههايى است كه در فواصل زمانى مساوى و منظمى جمع آورى شده شانه و بر اساس زماس زمان

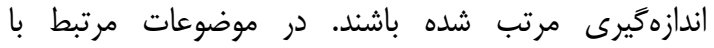

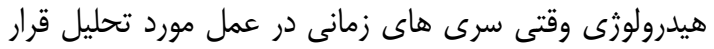

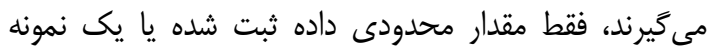

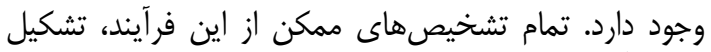

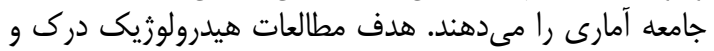

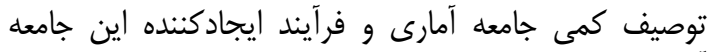

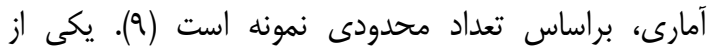

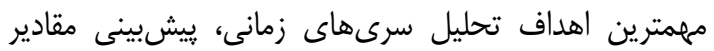

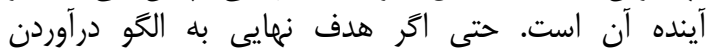

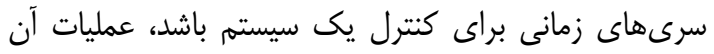

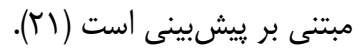

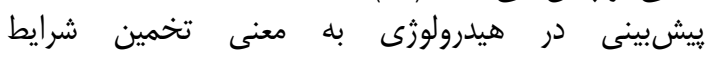
هيدرولوزيكى و هواشناسى در يكى بازه زمانى خاص مى باشند
MINITAB يس از آن، با استفاده از نرمافزار

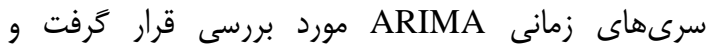

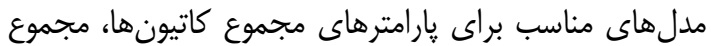

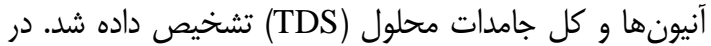

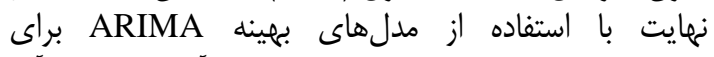

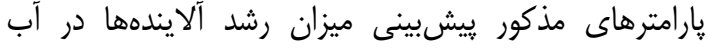
منطقه مورد مطالعه انجام شد. ملري

مدل L-THIA

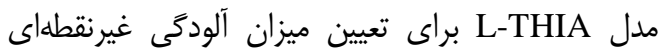

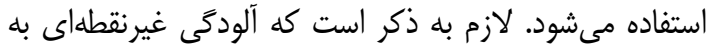

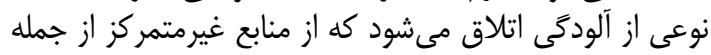

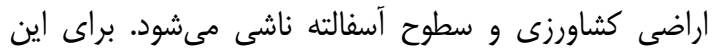

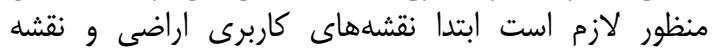

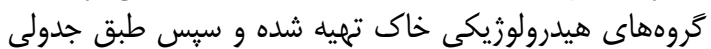

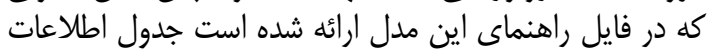

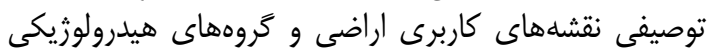

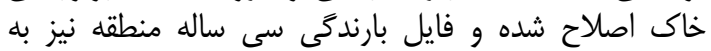

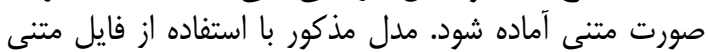

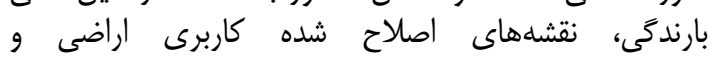

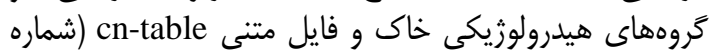

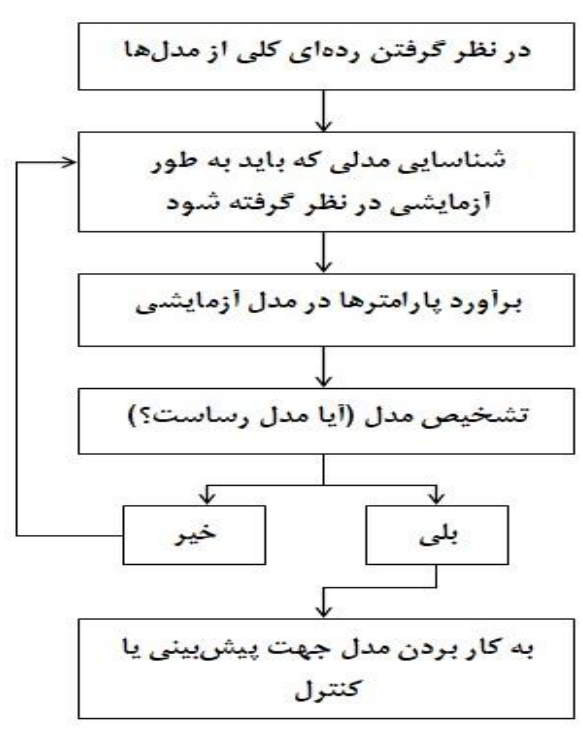

(1) ARIMA شكل r- مراحل مدلسازى

Figure 2. Steps of ARIMA modeling (16)

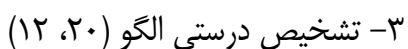

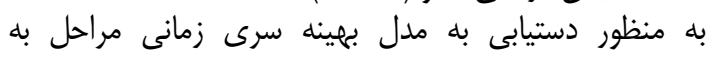
صورت شكل r طى مى مشود.

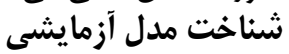

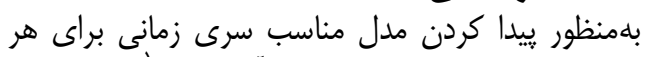

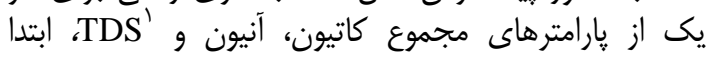

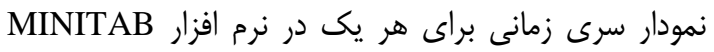

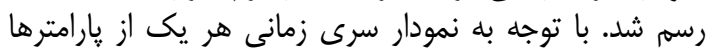

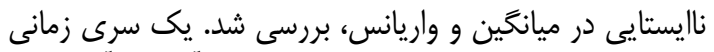

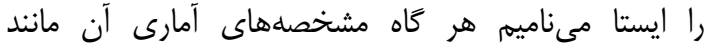

جهت ييش بينى سرىهاى زمانى لازم است كه رفتارهاى

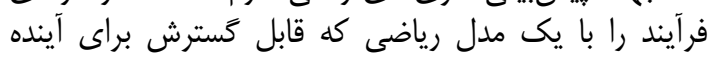

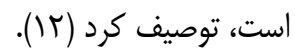
استراتزى الكَوسازى (مدل (باسازى)

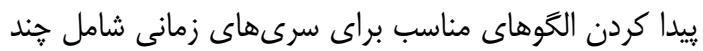
مرحله است كه بوسيله باكس و جنكينس در سال 1979 وضع نمانع شده است.

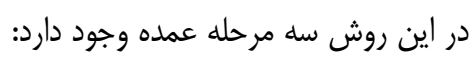

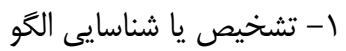

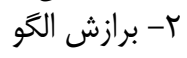


تعيين بهترين مدل موجود استفاده شد. خكّونكى محاسبه معيار آكائيك در رابطه مشخ مشص است.

$\operatorname{AIC}(q, p)=N \ln \left(\delta_{\Sigma}^{2}\right)+2(p+q)$

كه در آن N تعداد اطلاعات سرى زمانى و $\delta$ و واريانس

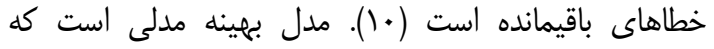
ميزان آكائيى كمترى را نشان دهد (سآ، بال). بيشبينى يس از تعيين مدل بهينه براى هر يكى از بارامترهاى مورد

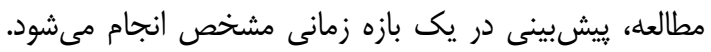

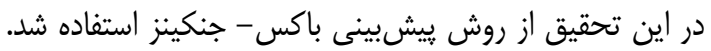

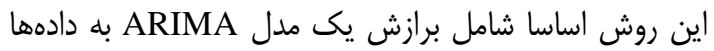

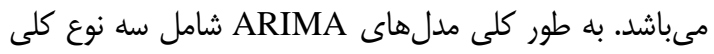

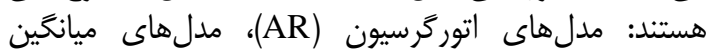

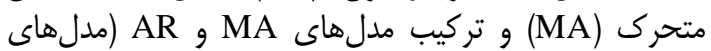
(ARIMA

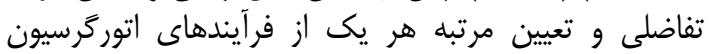
و ميانكين متحرك (MA) (AR)

\section{نتايج و بحث}

در اين تحقيق ابتدا مساحت كاربرىها در نقشه كاربرى

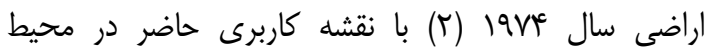
نرمافزار ArcMap10.2 مقايسه شد كه نتايج در جدارئ

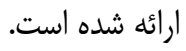

ميانغين و واريانس در طول زمان ثابت بماند. ابتدا به منظور

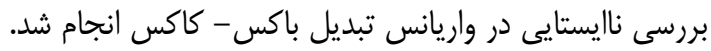

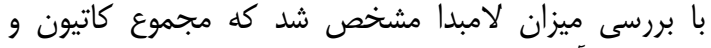

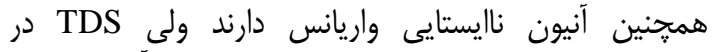

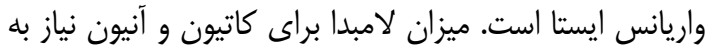

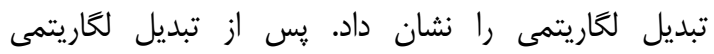

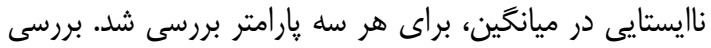

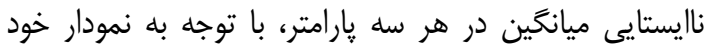

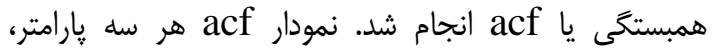

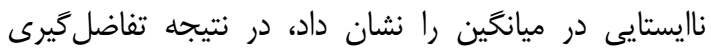

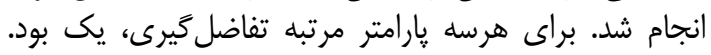

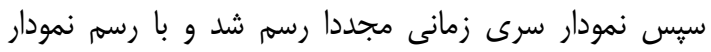

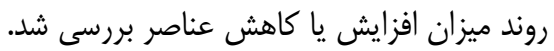

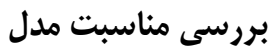

براى بررسى مناسب بودن مدن مدل از دول دور روش كه مكمل

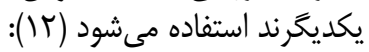

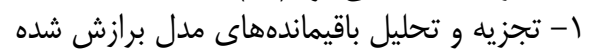

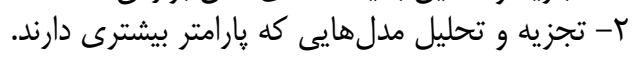

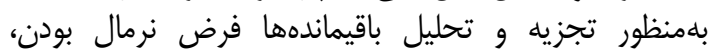

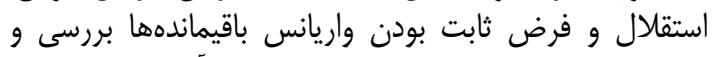

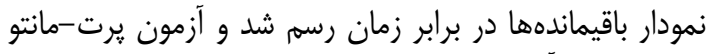

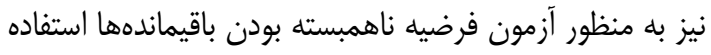

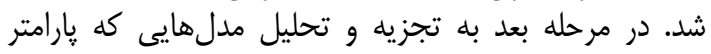

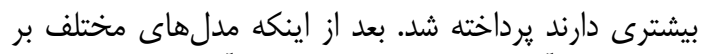
سرى زمانى آريما عبور داده شد از معيار آكائيك (AIC) براى مخلف

Table 1. Comparison the main land uses area in land use map of 1974 and 2017

جدول 1- مقايسه مساحت كاربرىهاى اصلى در نقشه كاربرى اراضى lave و و

\begin{tabular}{|c|c|c|c|c|}
\hline كاربرى اراضى VI • Y (درصد) & كاربرى اراضى l9Vץ (درصد) & كاربرى اراضى كنونى (ha) & (ha) I9VF كاربرى اراضى & نوع كاربرى اراضى \\
\hline $10 / 99$ & $1 . / 1$ & $119 \mp 9 / 4 F$ & V९qY/VG & كشاورزى \\
\hline$\Lambda r / \widetilde{\Lambda}$ & M/Q & GTVDV/FV & GVVDN/Fq & مرتع م \\
\hline$\cdot / 4 \wedge$ &.$/ \cdot 1$ & $r \Delta \omega / \bar{s}$. & $1 . / 48$ & مسكونى \\
\hline$\cdot / 4 V$ & س & $r \cdot r / \Delta q$ & $99 / .9$ & جاده \\
\hline $1 / 1 \Lambda$ & $\cdot / \Lambda$ & $9.9 / 90$ & $911 / \cdot v$ & ساير كاربرىها \\
\hline
\end{tabular}

شكله نشان داده شده است. در اين شكل ميزان افزايش

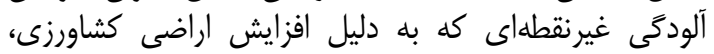

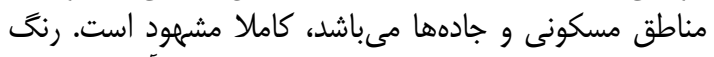

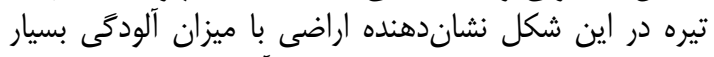

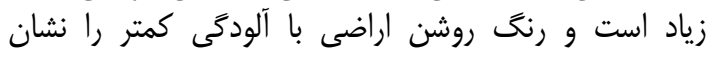
مىدهد.

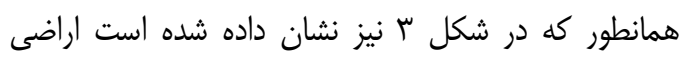

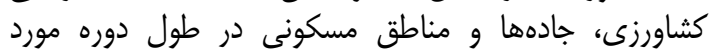
بررسى افزايش يافته اما وسعت مراتع كاهش يافت إفته است.

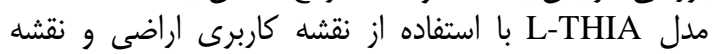

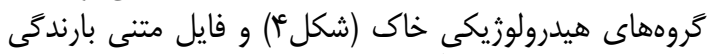

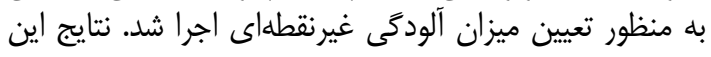

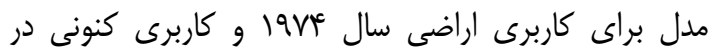



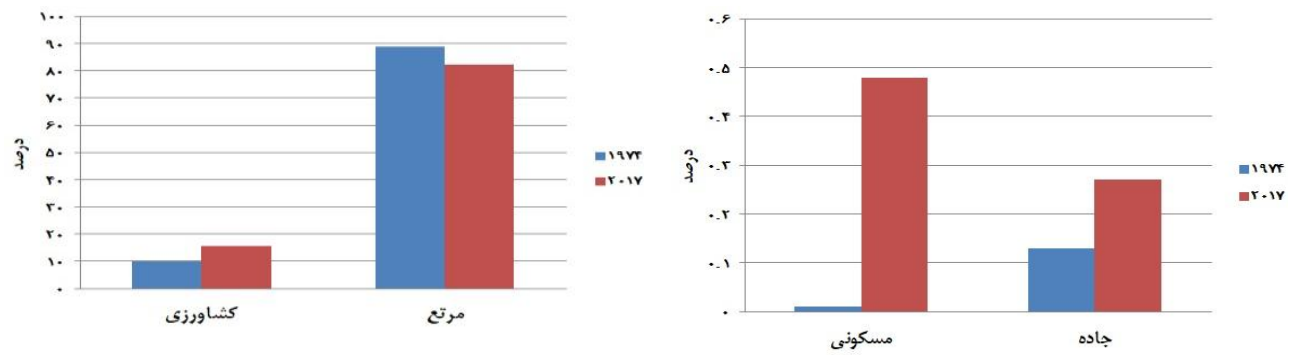

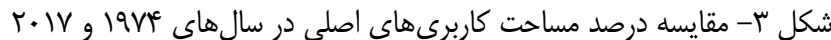

Figure 3. Comparison the percentage of the main land uses area in the years 1974 and 2017
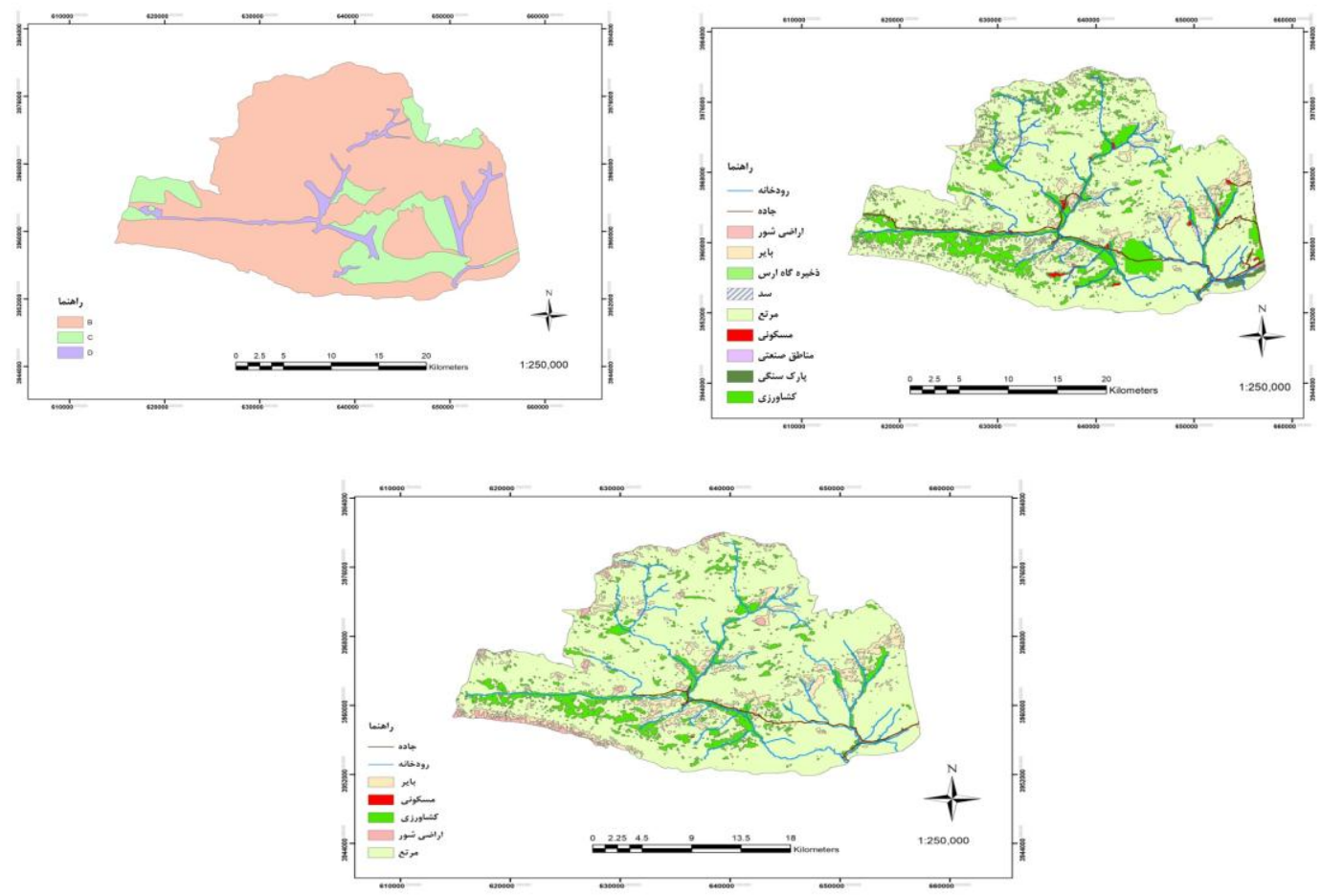

شكل ع- نقشه هاى ورودى مدل L-THIA : كاربرى اراضى IV +r (راست)، كاربرى اراضى (يإين)

Figure 4. The input maps of L-THIA model: land use of 2017 (right) and 1974 (left) and hydrological groups of Soil
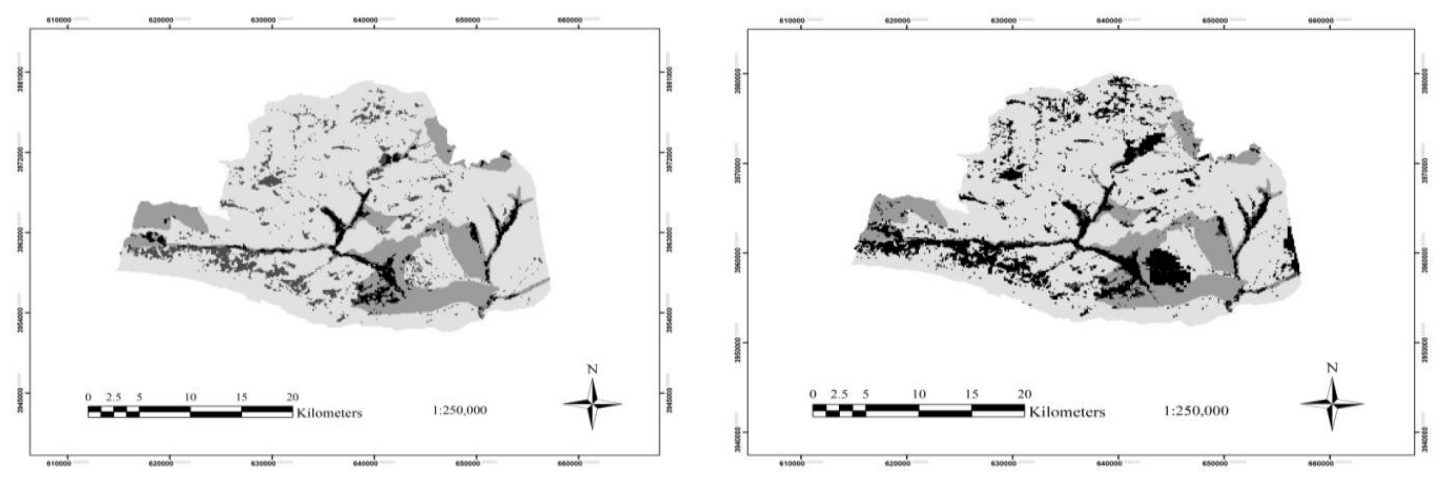

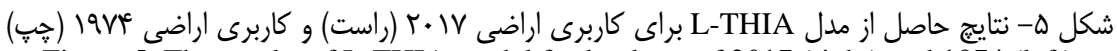

Figure 5. The results of L-THIA model for land use of 2017 (right) and 1974 (left) 

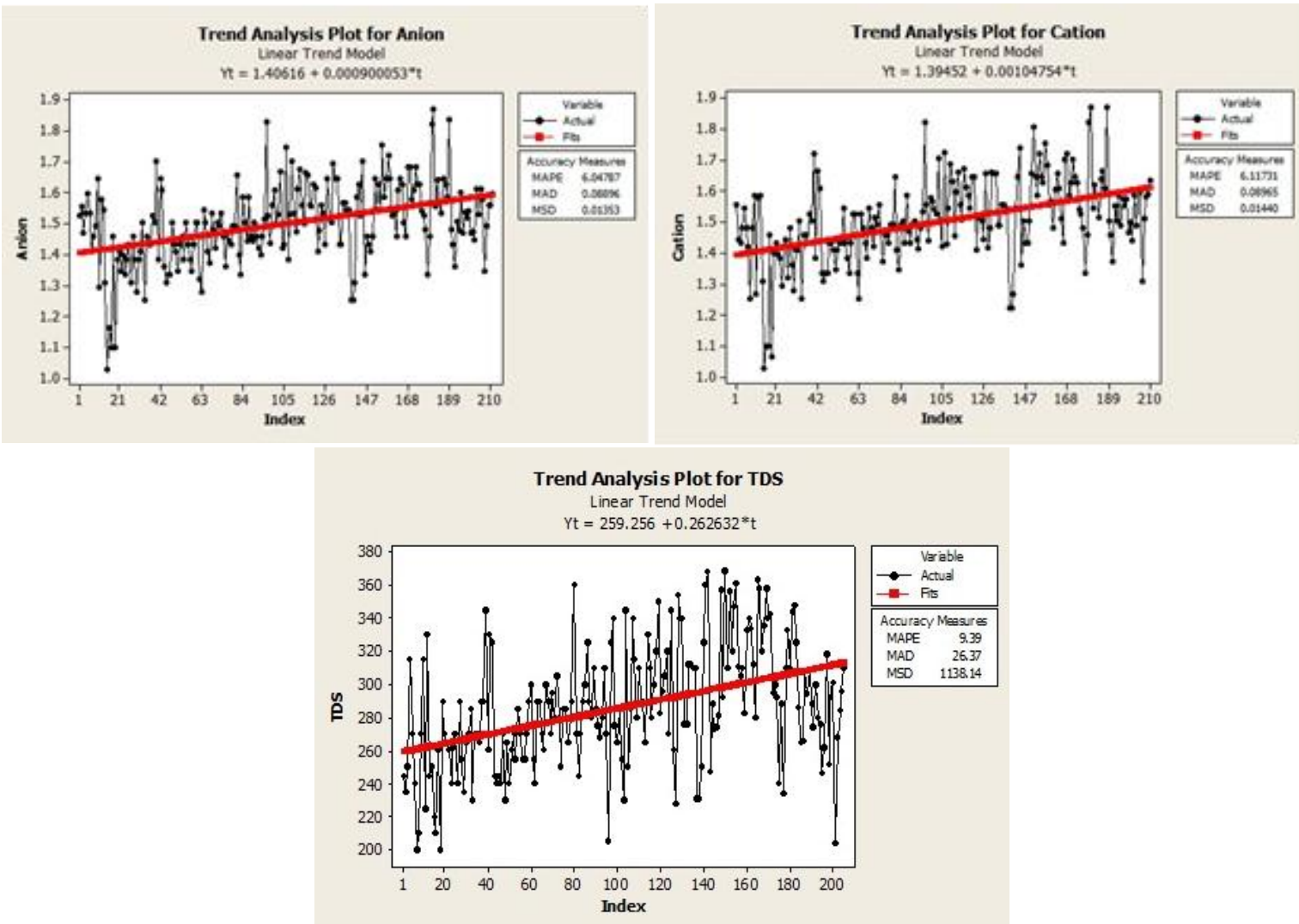

شكل צ- نمودار روند يارامترهاى مجموع كاتيون، آنيون و TDS

Figure 6. Diagrams of Trend Analysis Plot for total Cations, Anions and TDS

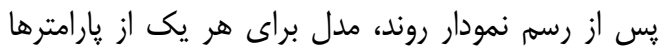

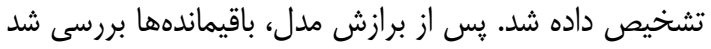

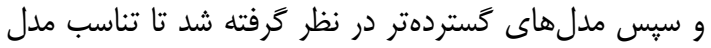

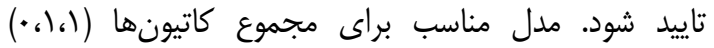
تشخيص داده شد.

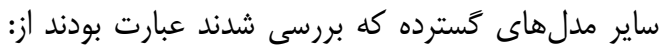

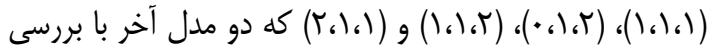

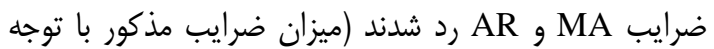

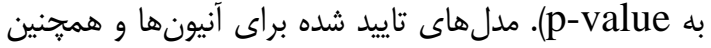

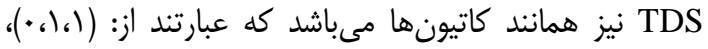

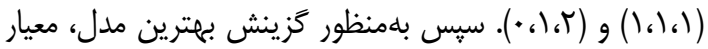

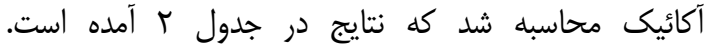

در اين شكل نقاط سياه نشان دهنده مقادير واقعى

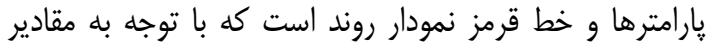

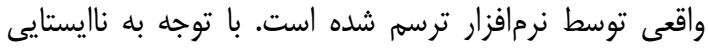

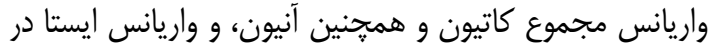

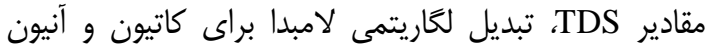

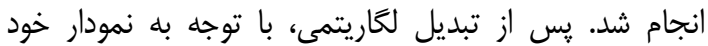

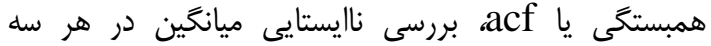

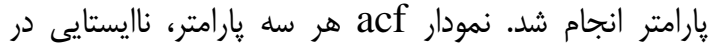

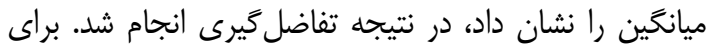

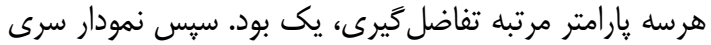

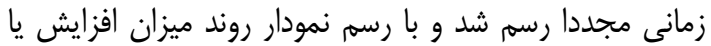
كاهش عناصر بررسى شد. نمودار سرى زمانى ونى و روند روند در شكل

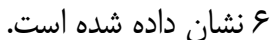

جدول r- مقايسه معيار آكائيك براى مدل بهينه كاتيونها، آنيونها و TDS Table 2. Comparison Akaike (AIC) criterion for optimal models of Cations, Anions and TDS

\begin{tabular}{|c|c|c|c|}
\hline معيار آكائيك TDS & معيار آكائيك آنيون & معيار آكائيى كاتيون & مدل ARIMA \\
\hline$-19 \% / \wedge V$ & - - $q \Psi / \wedge \vee$ & $-r \Lambda \Delta / r V$ & مدل ( (•،1، (1) \\
\hline$-194 / 19$ & $\underline{-r q 4 / 9 q}$ & -rMN/gr & مدل r (1، (1،) \\
\hline$-19 \pi / T \Delta$ & $-r q \tau / q$. & $-{ }^{\prime} \wedge q / \mu$. & مدل ץ (••(T) \\
\hline
\end{tabular}


كاتيونها، آنيونها و TDS به ترتيب در شكلهاى ل، 1 و و 9

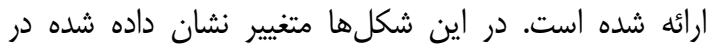

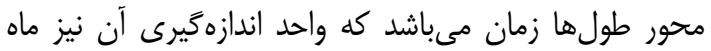

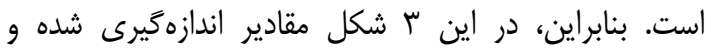

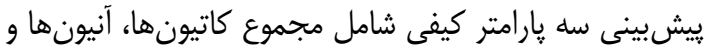
TDS

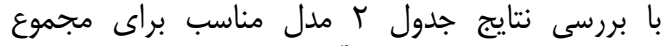

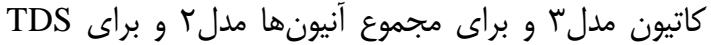

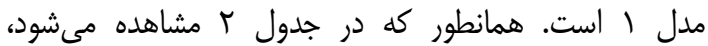
مدلهاى مذكور نشاندهنده كمترين مقادير آكائيك در درانطي

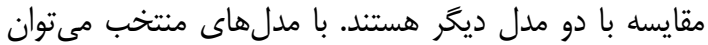

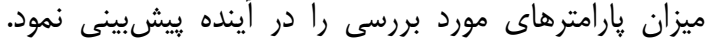

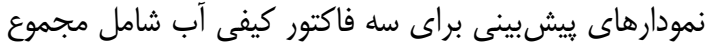
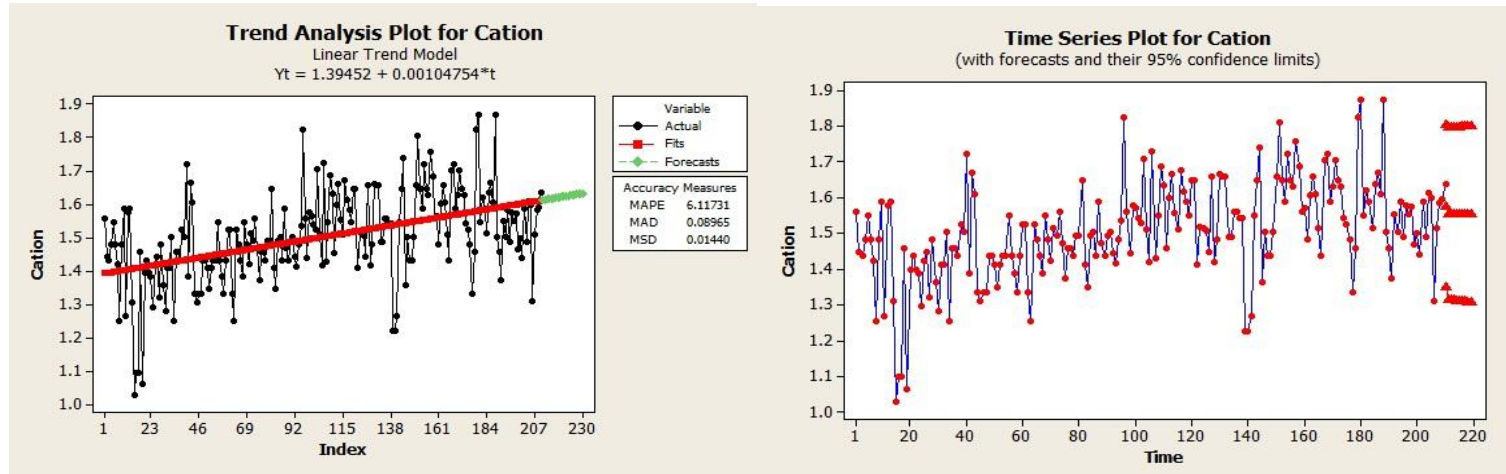

شكل V- نمودار يِيشبينى سرى زمانى و روند براى مجموع كاتيون

Figure 7. Forecasting diagrams of Trend Analysis and Time Series Plot for total Cations
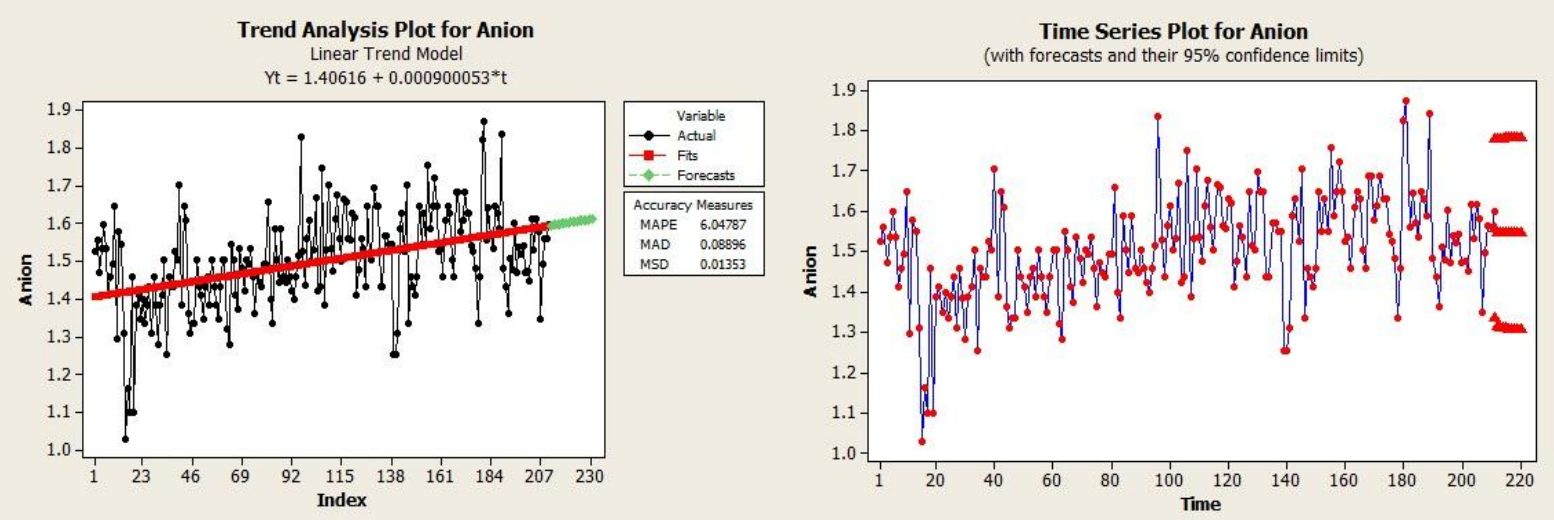

شكل ^- نمودار ييشبينى سرى زمانى و روند براى مجموع آنيون

Figure 8. Forecasting diagrams of Trend Analysis and Time Series Plot for total Anions
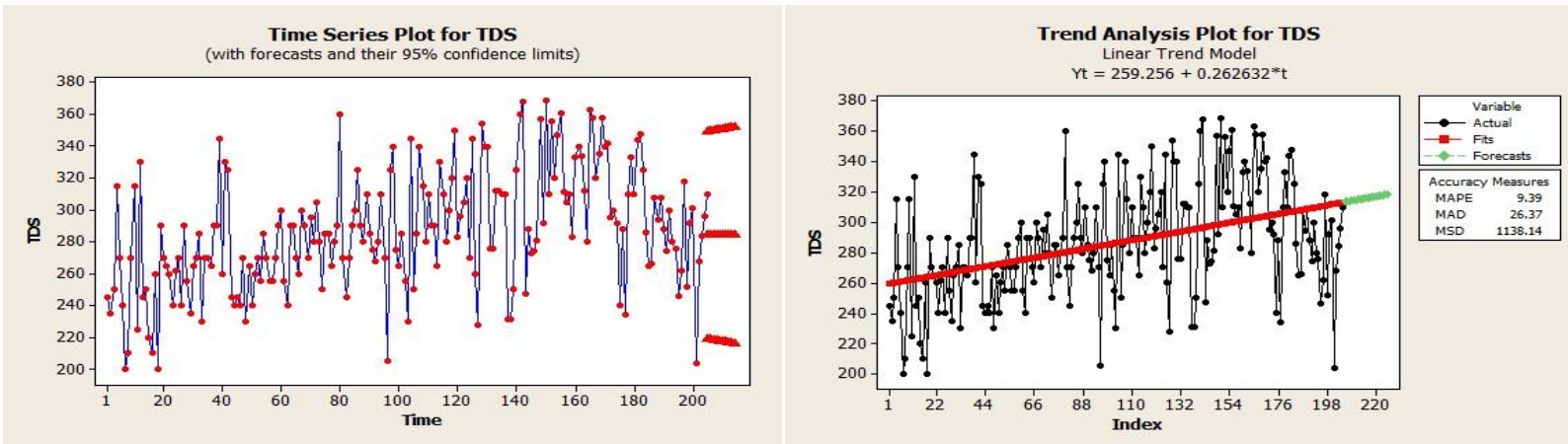

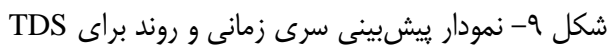

Figure 9. Forecasting diagrams of Trend Analysis and Time Series Plot for TDS 


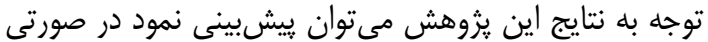

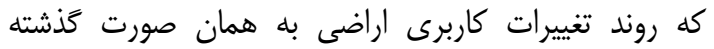
صورت كيرد، متاسفانه ميزان آلايندهائهاى كيفى آب آب رشان

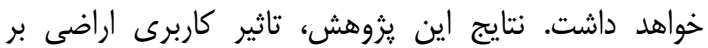

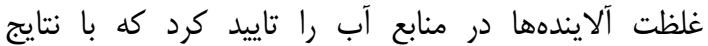
يزوهشهاى والتون و همكاران (YV)، خبرى و همكاران (I) (I)،

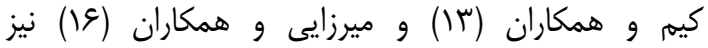

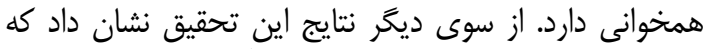

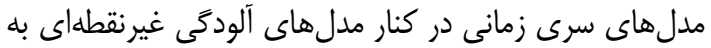

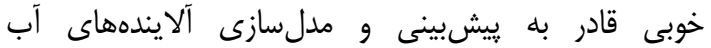

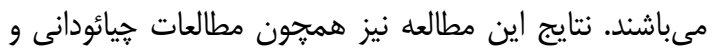

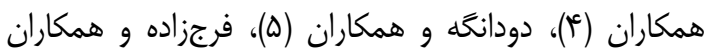

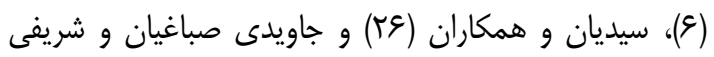

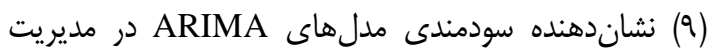

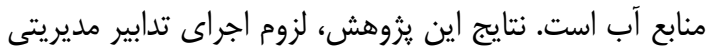

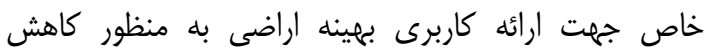

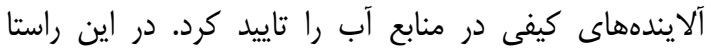

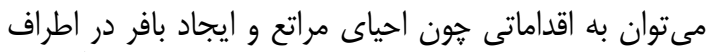

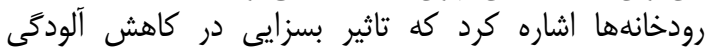
غيرنقطهاى دارند.
در اين تحقيق از مدل L-THIA و سرىهاى زمانى به

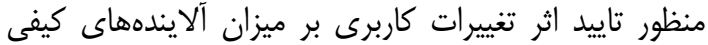

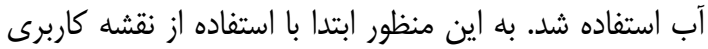

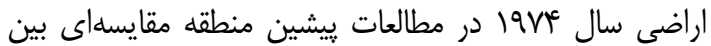

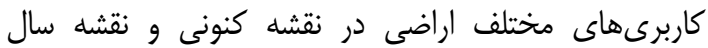


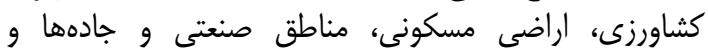

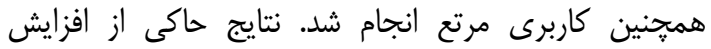

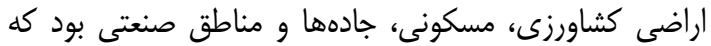

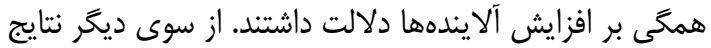

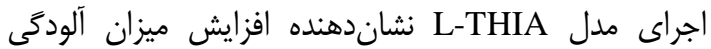

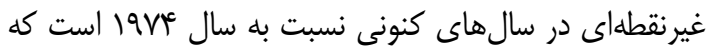

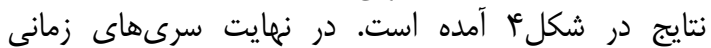
ARIMA كاتيونها، مجموع آنيونها و كل جاكل جامدات محلول (TDS)

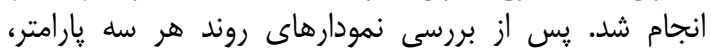

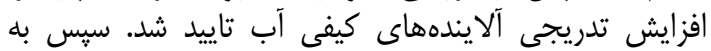

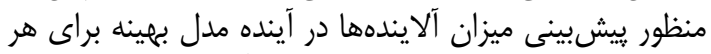

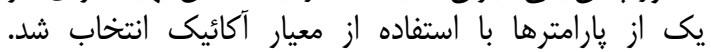

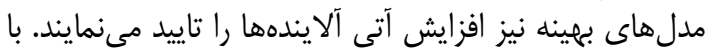

1. Afruzi, A and H. Zare Abyaneh. 2017. Groundwater Level Modeling and Forecasting using the T Series Models (Case Study: The Plains of Hamadan Province). Journal of Watershed Management Research, 8(15): 102-111 (In Persian).

2. Anonymous. 2012. Assessment of Economic, Social and Environmental effects in Hablehrood watershed. 342 pp (In Persian).

3. Babamiri, O., H. Nowzari and S. Maroofi. 2017. Potential Evapotranspiration Estimation using Stochastic Time Series Models (Case Study: Tabriz). Journal of Watershed Management Research, 8(15): 137-146 (In Persian).

4. Chiaudani, A., D.D. Curzio, W. Palmucci, A. Pasculli, M. Polemio and S. Rusi. 2017. Statistical and Fractal Approaches on Long Time-Series to Surface-Water/Groundwater Relationship Assessment: A Central Italy Alluvial Plain Case Study. Water, 9(11): 850, doi: 10.3390/w9110850.

5. Dodangeh, S., J. Abedi Koupai and A. Gohari. 2012. Application of time series modeling to investigate future climatic parameters trend for water resources management purposes. Sciences and Technology Agricultural and Natural Resources, Water and Soil Sciences, 16(59): 59-74 (In Persian).

6. Farajzadeh, J., A. Fakheri Fard and S. Lotfi. 2014. Modeling of monthly rainfall and runoff of Urmia Lake Basin using "feed-forward neural network" and "time series analysis" model. Water Resources and Industry, 7(8): 38-48.

7. Faryadi, S., K. Shahedi and M. Nabatpoor. 2012. Investigation of Water Quality Parameters in Tadjan River using Multivariate Statistical Techniques. Journal of Watershed Management Research, 3(6): 75-92 (In Persian).

8. Han, P., P.X. Wang, S.Y. Zhang and D.H. Zhu. 2010. Drought forecasting based on the remote sensing data using ARIMA models. Mathematical and Computer Modelling, 51: 1398-1403.

9. Javidi Sabbaghian, R. and M. Sharifi. 2009. Random modeling application in river flow simulation and estimation of mean annual river discharge by time series analysis. International Conference on Water Resources (ICWR). 9 pp (In Persian).

10. Khazaei, M. and M.R. Mirzaei. 2013. Comparison prediction performance of monthly discharge using ANN and time series. Watershed Engineering and Management, 2(2): 74-82 (In Persian).

11. Khebri, Z., F. Nejadkoorki and H. Sodaie Zadeh. 2015. The relationship between land use vector parameters and river water quality using GIS (Case study: Zayandeh rood river). RS \& GIS for Natural Resources, 6(1): 79-88 (In Persian).

12. Khorrami, M. and A. Bozorgnia. 2007. Time Series Analysis with MINITAB 14. Sokhangostar, Mashhad, Iran, 352 pp (In Persian).

13. Kim, Y., B.A. Engel, K.J. Lim, V. Larson and B. Duncan. 2002. Runoff Impacts of Land-Use Change in Indian River Lagoon Watershed. Journal of Hydrologic Engineering, 245-251. 
14. Koomen, E., J. Stillwell, A. Bakema and H.J. Scholten. 2007. Modeling land use change, progress and application. Springer, the Netherlands, $410 \mathrm{pp}$.

15. Li, Z., X. Deng, F. Wu and S.S. Hasan. 2015. Scenario analysis for water resources in response to land use change in the middle and upper reaches of the Heihe River Basin. Sustainability, 7: 3086-3108.

16. Mirzaei, M, E. Solgi, A. Salman Mahiny. 2017. Modeling of Non-Point Source Pollution by LongTerm Hydrologic Impact Assessment (L-THIA) (Case Study: Zayandehrood Watershed) in 2015. Archives of Hygiene Sciences, 6(2): 196-205.

17. Mohammadi, M., A. Kavian and L. Gholami. 2017. Simulation of Discharge and Nitrate in Tallar Basin using SWAT Model. Journal of Watershed Management Research, 8(15): 45-60 (In Persian).

18. Moshkani, M.R. 1992. Time series analysis: forcasting and control. Shahid Beheshti university of Tehran, Tehran, Iran, 424 pp (In Persian).

19. Munafo, M., G. Cecchi, F. Baiocco and L. Mancini. 2005. River pollution from non-point sources: a new simplified method of assessment. Environmental Management, 77: 93-98.

20. Niroomand, H.A. 1999. Time series analysis. Ferdowsi university of Mashhad, Mashhad, Iran, 404 pp (In Persian).

21. Niroomand, H.A. 2007. Time series analysis. Univariate and Multivariate Methods. Ferdowsi university of Mashhad, Mashhad, Iran, $586 \mathrm{pp}$ (In Persian).

22. Nury, A.H., K. Hasan and J.B. Alam. 2017. Comparative study of wavelet-ARIMA and wavelet-ANN models for temperature time series data in northeastern Bangladesh. Journal of King Saud University - Science, 29: 47-61.

23. Oliveira, J.P., J.L. Steffen and P. Cheung. 2017. Parameter Estimation of Seasonal ARIMA Models for Water Demand Forecasting using the Harmony Search Algorithm. Procedia Engineering, 186: 177-185.

24. Pirzadeh, B., M. Afsari, S.A. Hashemi Monfared and A.A. Ghaderi. 2017. Generating Artificial Water Quality Data for No-Trend Parameters in Reservoirs (Chahnimeh No.1 in Sistan). Iran-Water Resources Research, 13(2): 226-232 (In Persian).

25. Salajegheh, A., S. Razavizadeh, N. Khorasani, M. Hamidifar and S. Salajegheh. 2011. Land use changes and its effects on water quality (case study: Karkheh watershed). Environmental Studies, 37(58): 22-26.

26. Seyediyan, M., M. Soleymani and M. Kashani. 2014. Forecasting of water discharge using data analysis and time series. Ecohidrology, 1(3): 167-179 (In Persian).

27. Walton, R.S. and H.M. Hunter. 2009. Isolating the water quality response of multiple land use from stream monitoring data through model calibration. Journal of Hydrology, 378: 29-45. 


\title{
Prediction of the Type and Amount of Surface Water Pollutants using Time Series Models (ARIMA) and L-THIA Model (Case Study: Namrood Sub-Basin, Hablehrood Watershed)
}

\author{
Zahra Pasandidehfard ${ }^{1}$, Ali Reza Mikaeili Tabrizi ${ }^{2}$, Abolfazl Mosaedi ${ }^{3}$ and Hasan Rezaei ${ }^{4}$ \\ 1- Ph.D. Student of Environmental Sciences- Land use Planning, Gorgan University of Agricultural and Natural \\ Resources (Corresponding author: Zahra_pasandidehfard@yahoo.com) \\ 2 and 4- Associate Professor and Assistant Professor, Faculty of Environment, Gorgan University of Agricultural \\ Sciences and Natural Resources \\ 3- Professor, Faculty of Natural Resources and Environment, Ferdowsi University of Mashhad \\ Received: January 25, 2018 \\ Accepted: August 27, 2018
}

\begin{abstract}
Due to the important role of non-point source pollution in water resources management, in this study time series modeling was applied to forecast water quality parameters and L-THIA model (one type of non-point source pollution models) was applied to estimate water pollutants. The purpose of this study was to compare results of L-THIA model and ARIMA models in Namrood sub-basin located in the Hablehrood watershed. At first, land use changes were studied from the years 1974 till 2017 that showed increase in agricultural lands and expansion of cities and roads. Then, using L-THIA model for both land use categories, the amount of pollutant and the volume of runoff were calculated that showed high growth. In the end, using ARIMA models were estimated water quality parameters for 30 years. Among the different ARIMA models, a model with a lowest error and akaike (AIC) criterion was selected as an optimal model for TDS, total of cations and anions. Desirable models for TDS, total of cations and anions were $(0,1,1),(1,1,2)$ and $(1,1,1)$, respectively. The end, diagrams of Trend Analysis and Time Series were performed for three parameters that indicated high growth in amount of pollutant. The results showed efficiency of time series modeling in water resources studies in order to forecast water quality parameters.
\end{abstract}

Keywords: L-THIA model, Non-point source pollution, Runoff, Time series models, Water quality parameters 